\title{
Measurement of the Characteristics of TIDs Using Small and Regional Networks of GPS Receivers during the Campaign of 17-30 July of 2008
}

\author{
Cesar E. Valladares ${ }^{1}$ and Matthew A. Hei ${ }^{2}$ \\ ${ }^{1}$ Institute for Scientific Research, Boston College, Chestnut Hill, MA 02467, USA \\ ${ }^{2}$ Plasma Physics Division, Naval Research Laboratory, Washington, DC 20375, USA \\ Correspondence should be addressed to Cesar E. Valladares, cesar.valladares@bc.edu
}

Received 4 November 2011; Accepted 17 February 2012

Academic Editor: Y. Sahai

Copyright (C) 2012 C. E. Valladares and M. A. Hei. This is an open access article distributed under the Creative Commons Attribution License, which permits unrestricted use, distribution, and reproduction in any medium, provided the original work is properly cited.

\begin{abstract}
This scientific report presents the results of a dedicated experiment that was conducted within the framework of the Low-latitude ionospheric Sensor Network (LISN) observatory to measure the characteristics of medium-scale (hundreds of km) Traveling Ionospheric Disturbances (TIDs) as they transit through the low-latitude ionosphere. A small array of 3 GPS receivers separated by $4-5 \mathrm{~km}$ placed in a triangular configuration was installed near Huancayo in Peru possessing several characteristics of a radiointerferometer. During the campaign days, 17-30 July 2008, TIDs were observed daily. On July 20, 2008 between 22 and 24 UT several TIDs moved across the small array of GPS receivers with a velocity near $130 \mathrm{~m} / \mathrm{s}$, were directed northward and had wavelengths close to $450 \mathrm{~km}$. Other GPS receivers that were operating hundreds of $\mathrm{km}$ away from Huancayo show also similar TEC traces and provide a phase velocity equal to $150 \mathrm{~m} / \mathrm{s}$. This value was measured using the GPS at Piura, Cuzco and Huancayo. Based on this positive result, we conclude that small and/or regional arrays of GPS receivers can be used at low latitudes to study the role that gravity waves may have on seeding plasma bubbles.
\end{abstract}

\section{Introduction}

Traveling ionospheric disturbances (TIDs) are the ionospheric manifestation of neutral density oscillations called atmospheric gravity waves (AGWs or GWs). AGWs originate in the troposphere or stratosphere, and often propagate to F-region heights, where they induce oscillations in the plasma density via ion-neutral coupling. When AGW winds drive plasma along magnetic field lines via ion-neutral collisions, "classical" TIDs result [1-3]. However, at night the electrodynamics are favorable to the production of "nonclassical" or "electrodynamic" TIDs, such that vertical movement of the F-layer results [4-6]. TIDs that reach F-region altitudes are divided into two classes: mediumscale (MSTIDs) and large-scale (LSTIDs). MSTIDs primarily originate from tropospheric weather systems and mountain turbulence; depending on background wind conditions and other factors, these may propagate in any direction. LSTIDs are produced by auroral energy injection during geomagnetic storms. They typically propagate meridionally from polar regions towards the equator. AGWs have a wide range of observed wavelengths and propagation velocities, frequently having horizontal wavelengths of up to hundreds of kilometers and speeds of hundreds of meters per second. As they propagate from lower altitudes, their amplitudes grow exponentially. At mid-latitudes, AGWs have been observed to have preferred propagation azimuths that change with season [7]; it is logical to assume that similar seasonal patterns exist at low latitudes.

Gravity waves are widely believed to play a critical role in seeding the development of equatorial plasma depletions in the nighttime low-latitude ionosphere. Current understanding holds that both favorable ionospheric conditions [8] and an initial seed perturbation on the bottomside are necessary to create rising depletion channels. If both are present, the Rayleigh-Taylor instability creates intense polarization 
electric fields within the perturbations, causing the depleted plasma inside to $E \times B$ drift upward into the topside $[9,10]$. Gravity waves, [11], as well as penetration electric fields, dynamics associated with the post-sunset vortex [12], and E-region structure mapped to F-region altitudes along magnetic field lines [13] have all been cited as possible seeding mechanisms.

To unravel the importance of the gravity wave seeding mechanism, it is important to observe and characterize both early-evening gravity waves and the equatorial plasma depletions seen later in the evening. However, most current observing techniques have severe limitations. Ionosondes can measure the GW vertical period, but they are not sensitive to other parameters. A steerable-beam radar such as Altair can measure the large-scale zonal wavelength and amplitude, but the GW must be traveling roughly orthogonal to the radar beam to be detected. Phased-array radar systems such as the Japanese MU system [14, 15] are sensitive to GW horizontal wavelength, propagation azimuth, and amplitude, but these systems are expensive to deploy and operate. OI $(557.7 \mathrm{~nm}$, emission altitude $\sim 96 \mathrm{~km})$ and $\mathrm{OH}$ band $(720-910 \mathrm{~nm}, \sim 86 \mathrm{~km})$ airglow imagers can measure GW horizontal wavelengths, propagation azimuths, and amplitudes, but at altitudes far below the bottomside [16, 17]. OI (630 nm emission) optical measurements have been used to characterize the seasonal and solar cycle variability of MSTIDs [18, 19]. OI has an emission layer between roughly 200-300 km, which is very close to the bottomside [20]. But even here, airglow imagers operate only during the nighttime and require good seeing conditions (no clouds) to operate.

The radio beacon technique [7, 21-23] overcomes many limitations of other observation methods, enabling simultaneous characterization of key GW parameters. Here, satellite radio beacon signals are measured by stationary ground receivers; TIDs are evident as fluctuations in total electron content (TEC) derived from the beacon signals [24]. Although TEC is an integrated quantity, various processes, including wind-filtering [7] and breaking [25], prevent AGWs from penetrating far into the thermosphere. Observations [26] and model results [27] have shown that most TIDs do not rise above $300 \mathrm{~km}$. Thus, the bulk of the TEC perturbations occur on the F-region bottomside and the upper E-region. Moreover, after the E-region decays in the evening, TEC fluctuations become highly localized in the bottomside region. Thus, while radio beacon TEC measurements are integrated over the whole altitude range of the ionosphere, the narrow vertical localization of GW-induced TEC fluctuations makes this technique a powerful tool for studying early-evening TIDs. In the radio beacon technique, also known as radio-interferometry, phase differences measured at the various stations are used to determine TID velocity, propagation azimuth, and amplitude.

Recently, the radio-interferometric technique has been adapted for use with GPS satellites [28-30]. This new innovation makes it possible to utilize inexpensive, easily deployed GPS receivers to study gravity waves at a wide variety of locations. The large number of GPS satellites in orbit makes it possible to continuously monitor the bottomside region over a given receiver array.

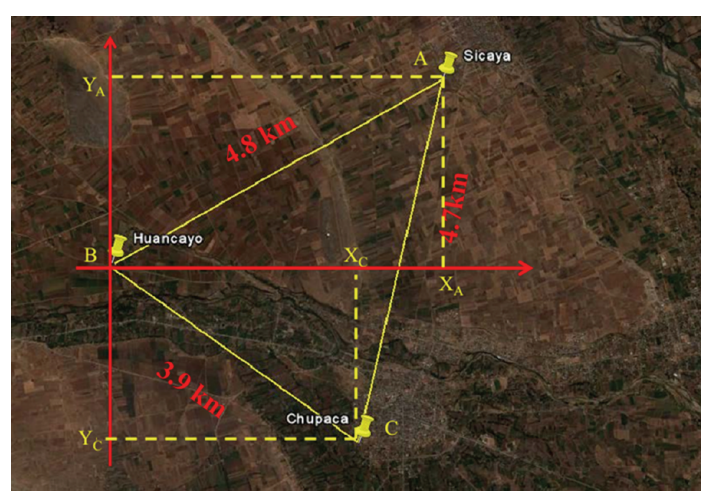

FIGURE 1: Geographic location of the Huancayo radio interferometer test array.

This paper describes measurements observed with a three-station array of GPS receivers that were located near Huancayo, Peru. These receivers constitute the first lowlatitude radio-interferometer array dedicated to studying TIDs. The hardware layout and the analysis technique, that was used to derive the TEC perturbation associated with the TIDs, are described in the next section. The phase velocities, scale sizes, and the direction of motion of the TIDs calculated using the SADM-GPS methodology are presented in Section 3. The TIDs wave characteristics derived using a cross-correlation method are introduced in Section 4. The cross-correlation method was also applied to TEC measurements recorded by other low-latitude ionosphere sensor network (LISN, http://lisn.igp.gob.pe/) GPS receivers that are operating hundreds of kms apart from Huancayo. The results of this analysis are described in Section 5. Discussion and conclusions follow in Sections 6 and 7.

\section{Observations}

Since the late 90s several publications have reported the characteristics of mid-latitude GWs using the GPS radiointerferometry (GPS-RI) method [28, 30]. Based on this success, we conducted a campaign in South America aiming to characterize the local time, duration, and wave properties of GWs that are transiting near the magnetic equator in the Peruvian sector. We installed two GPS receivers near the city of Huancayo $\left(12.042^{\circ} \mathrm{S} ; 75.321^{\circ} \mathrm{W}\right)$ due to its proximity to the magnetic equator, the existence of a permanent GPS receiver in that city and the presence of nearby towns. The additional GPS receivers were temporarily installed, one at the town of Chupaca $\left(12.061^{\circ} \mathrm{S} ; 75.292^{\circ} \mathrm{W}\right)$ and the other at Sicaya $\left(12.021^{\circ} \mathrm{S} ; 75.282^{\circ} \mathrm{W}\right)$. All 3 GPS receivers of the small network of GPS receivers consist of GSV4004B Novatel GPS using geodetic antennas. Similarly, Novatel GPS receivers were also installed in the cities of Piura and Cuzco. However, the receiver in Iquitos consists of a CRS1000 LEICA GPS that uses a choke ring antenna. Figure 1 indicates the geographic locations of the three sites, where GPS receivers operated between July 17-30, 2008 and July 30. As indicated in Figure 1, the distance between GPS receivers was between 3.9 and $4.8 \mathrm{~km}$. They were distributed forming an isosceles 

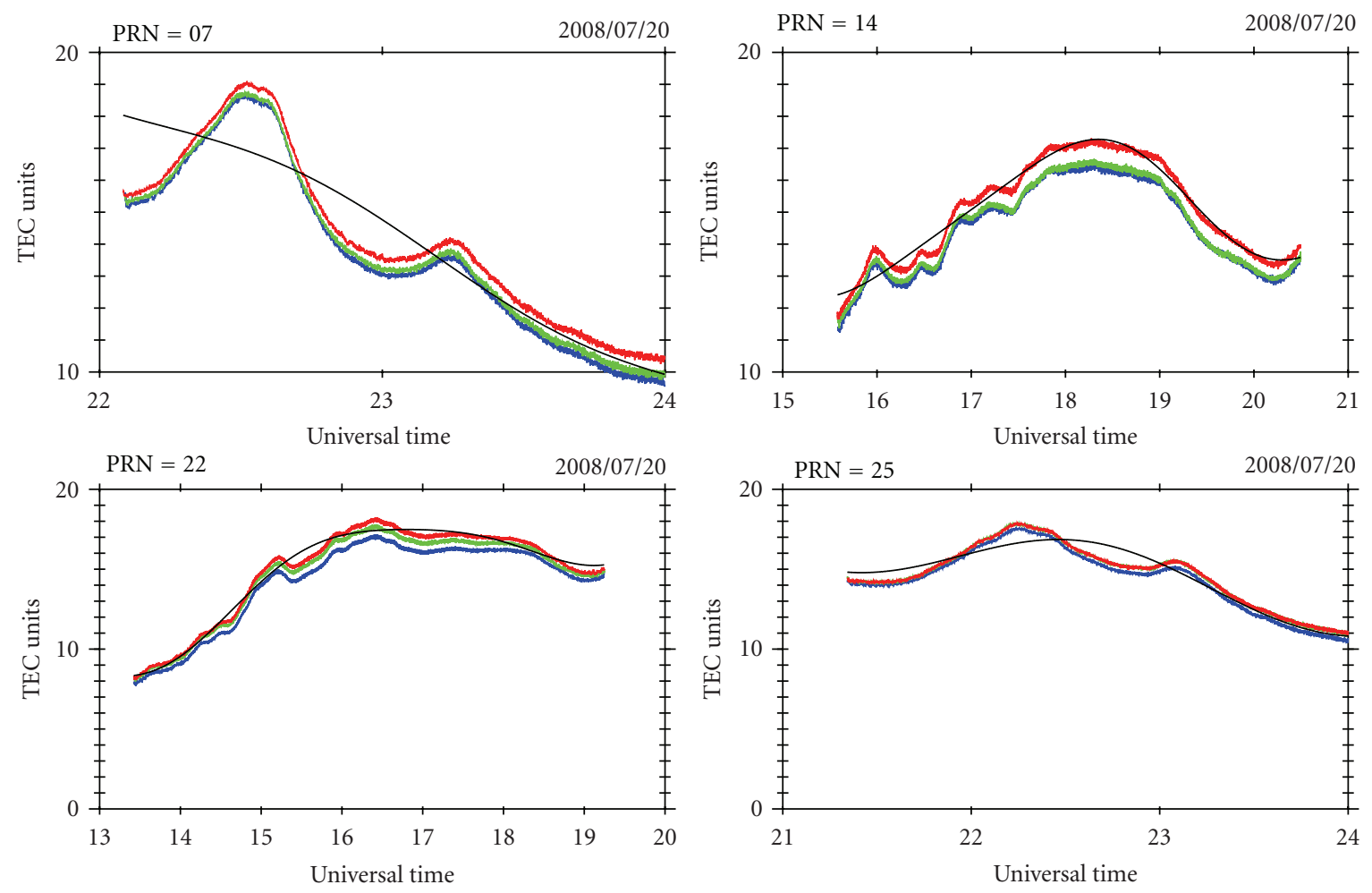

FIGURE 2: TEC versus UT measured by the Huancayo GPS receivers; red, green, and blue traces represent TEC values from the three receivers; black lines represent the estimated background/unperturbed TEC values. Each of the four panels represents signals from one of four GPS satellites (PRN $=07,14,22$, and 25).

triangle to provide good estimates of the phase velocity as a function of both latitude and longitude. Due to the relatively short distance between receivers and the relatively large phase velocity of GWs that can vary between 100 and $500 \mathrm{~m} / \mathrm{s}$ [31], we opted to sample the phase and group delay of both $\mathrm{L} 1$ and L2 frequencies once per second $(1 \mathrm{~Hz})$.

Each of the four frames of Figure 2 present TEC values measured by all 3 GPS receivers that operated near Huancayo on July 20,2008 . The TEC values from different receivers have been color coded to differentiate them and to indicate their similarity. The TEC values were computed following the standard procedure that involves using the differential phase and pseudorange values of both L1 and L2 frequency signals [32] and leveling the differential phase to the average differential code values to eliminate phase ambiguity. The absolute values of TEC were then calculated by introducing the differential satellite biases published by the University of Bern and calculating the receiver bias by a minimization process of the TEC variability between 0200 and 0600 local time. The thin black line, displayed in each of the 4 frames of Figure 2, indicates an estimate of the background or unperturbed TEC values. This estimate takes into account the typical daily variability of the TEC values that is a function of the local time variability produced by solar EUV radiation, changes in tides, presence of external electric fields, and more importantly the TEC variability introduced by the motion of the GPS satellites that continuously samples different latitudes and longitudes. These background TEC values are used to estimate the TEC perturbation, or in other words, derive the contribution produced by the passage of the gravity waves. The TEC curves corresponding to each satellite pass were fitted to a 4th-order-polynomial to filter out the diurnal variability and to remove spatial changes in TEC due to the GPS satellite motion and to evaluate TEC perturbations (TECP) containing time scales of 3 hours or less. Similar analysis was conducted by Valladares et al. [24] to estimate the TEC perturbation attributed to the passage of large-scale gravity waves. Equatorial plasma bubbles can also produce significant perturbations in the TEC traces. However, their effect consists of rapid and deep negative excursions of the TEC traces, commonly called TEC depletions. To avoid any possible erroneous identification, the GW/TID campaign was carried out during a period of minimum bubble activity, in which no TEC depletions were observed. As shown in the following figures, all the analysis methods are based on the difference between the measured and the estimated background "unperturbed" TEC values. This differential value is called TEC perturbations (TECP).

Figure 3 shows TECP values measured on 3 different days. The top panel, corresponding to July 17,2008 , displays perturbations that reach 1 TEC unit between 20 and 22 UT. Smaller amplitude TEC perturbations $( \pm 0.4$ TEC units) are observed between 13 and 19 UT. The noise level in this and other panels is about \pm 0.2 TEC units. The TEC perturbations of the center panel (July 20, 2008) exceed 1 TEC unit between 22 and 24 UT and exhibit moderate levels ( \pm 0.6 TEC units) 

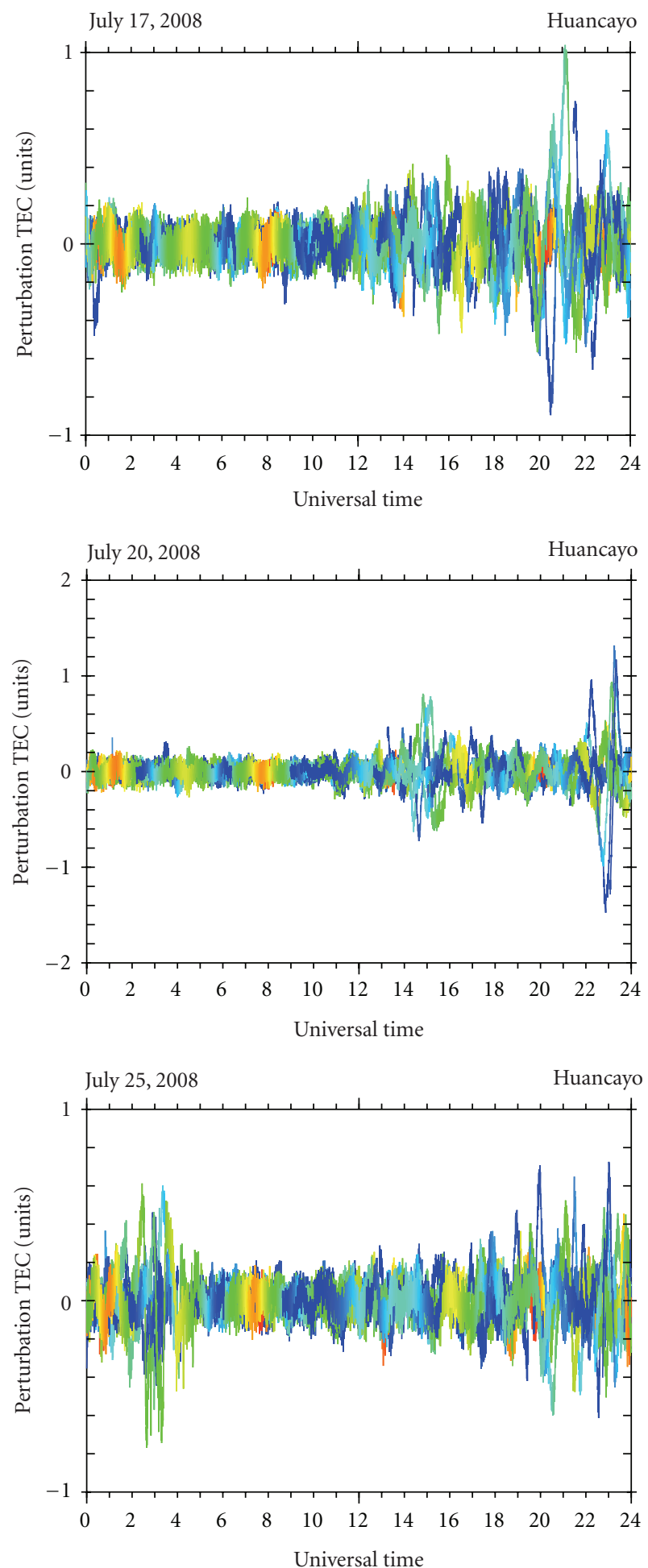

FIGURE 3: TECP versus UT at Huancayo for three days in July. Data from various GPS satellite signals are plotted; each satellite is distinguished by a different color.

of TEC perturbations between 14 and 16 UT. Note the different amplitude scale that is used in this panel. The bottom panel corresponding to July 15, 2008 depicts two large patches with significant levels of TEC perturbations. The first occurs during early evening hours (00-04 UT) and the second in the afternoon hours (19-24 UT). In summary, during the campaign TEC perturbations are observed preferentially a few hours before sunset (24 UT). A second interval of TEC perturbations occurs at different times of the day. 


\section{Analysis of TID Characteristics Using SADM-GPS Algorithm}

We have calculated the TIDs horizontal phase velocity $V_{h}(t)$ and the azimuthal propagation direction of the phase front $\alpha(t)$ using the statistical angle of arrival and doppler method for GPS interferometry (SADM-GPS) developed by Afraimovich et al. [28-30]. This method assumes that the ionosphere changes in a nondispersive manner due to the passage of the TID or a packet of TIDs. Under this assumption, Afraimovich et al. [28, 30] calculated the velocity and direction of propagation of mid-latitude TID packets during quiet ionospheric conditions. The SADM-GPS represents the TEC perturbations by the form: $I(x, y, t)=\delta(t) \sin \left(\Omega t-k_{x} x-\right.$ $\left.k_{y} y+\varphi_{0}\right)$. This expression implies that a monochromatic or a largely dominant TID propagates through the field-ofview of the small receiver network. For events when more than one TID is propagating through the measured volume, this representation will be invalid, and a spectral characterization of the TIDs is more appropriate. Using the equations proposed by Afraimovich et al. [28], it can be demonstrated that the TID wave parameters can be calculated using:

$$
\begin{gathered}
\alpha(t)=\arctan \left(\frac{I_{x}^{\prime}(t)}{I_{y}^{\prime}(t)}\right)=\arctan \left(\frac{G_{x}}{G_{y}}\right) \\
u_{x}(t)=\frac{I_{t}^{\prime}(t)}{I_{x}^{\prime}(t)} \\
u_{y}(t)=\frac{I_{t}^{\prime}(t)}{I_{y}^{\prime}(t)} \\
V_{h}(t)=u(t)+w_{x}(t) \sin \alpha(t)+w_{y}(t) \cos \alpha(t) \\
\frac{\left(u_{x}(t) u_{y}(t) \mid u_{y}^{2}(t)\right)}{(1 / 2}, \\
G_{y}=\frac{\left(X_{C}\left(\mathrm{TECP}_{B}-\mathrm{TECP}_{A}\right)-X_{A}\left(\mathrm{TECP}_{B}-\mathrm{TECP}_{C}\right)\right)}{X_{A} Y_{C}-X_{C} Y_{A}} \\
G_{x}=\frac{\left(Y_{A}\left(\mathrm{TECP}_{B}-\mathrm{TECP}_{C}\right)-Y_{C}\left(\mathrm{TECP}_{B}-\mathrm{TECP}_{A}\right)\right)}{X_{C} Y_{A}}
\end{gathered}
$$

where $I^{\prime} x(t), I^{\prime} y(t)$, and $I^{\prime} t(t)$ are the spatial and time derivatives of the TECP values. $u_{x}(t)$ and $u_{y}(t)$ are the propagation velocities of the phase front along the $x$-(east) and $y$-(north) axes in a frame of reference centered at the Huancayo station. $w_{x}(t)$ and $w_{y}(t)$ are the $x$ and $y$ projections of the sub-ionospheric intersection point velocity. Note that $G_{x}$ and $G_{y}$ symbols used in Figure 4 are equivalent to the $I^{\prime} x(t)$ and $I^{\prime} y(t)$ derivatives. As the velocity of the subionospheric intersection
$(80-100 \mathrm{~m} / \mathrm{s})$ is comparable to the speed of the GWs, it becomes imperative to subtract this effect from the estimated phase velocity $u(t)$. Equation (5) includes this correction in which the component of the satellite velocity in the direction perpendicular to the phase front is subtracted to determine the true horizontal velocity of the TID. The symbols $X_{A}, Y_{A}$, $X_{C}$, and $Y_{C}$ are defined in Figure 1 and correspond to the coordinate distances of Sicaya $(A)$ and Chupaca $(C)$ in a Cartesian system. To eliminate noise fluctuations containing periods less than $5 \mathrm{~min}$, a low-pass filter was applied to all TECP traces shown in subsequent figures.

Figure 4 presents the results of applying (1) through (7) to the TECP values measured on July 20, 2008 between 21 and 24 UT. This figure follows closely the format presented by Afraimovich et al. [28] in Figure 9. Panels (a) and (e) show the time derivative $\left(G_{t}\right)$ of the TECP measured at Huancayo from GPS satellites 13 and 25, respectively. Panels (b) and (f) display the east-west $\left(G_{x}\right)$ spatial derivative using a solid line, and the north-south $\left(G_{y}\right)$ spatial derivatives using dots. Note that $(6)\left(G_{x}\right)$ and $(7)\left(G_{y}\right)$ are a function of the TECP values measured by all 3 GPS receivers belonging to the small array. Panels (c) and (g) present the angle of the wave front, $\alpha(t)$, that was calculated using (1). It is noted that this angle is measured clockwise with respect to geographic north. Panels (d) and (h) introduce the phase velocity of the TID calculated using (5). The large scatter of the $\alpha(t)$ and $V_{h}(t)$ values is caused by the nonmonochromatic nature of the TECP values. However, the average lines in these frames indicate our best estimate of the TID phase velocity that varies between $3^{\circ}$ and $6^{\circ}$. The estimation of the horizontal velocity of the dominant TID falls between 107 and $143 \mathrm{~m} / \mathrm{s}$. Comparison of the $G_{t}$ traces for satellites 13 and 25 (panels (a) and (e)) show largely different magnitudes; this is due to the different look angle direction that varies between these two GPS satellites. GPS satellite 13 passed $200 \mathrm{~km}$ away from Huancayo and GPS 25 went over Huancayo almost overhead. Mercier and Jacobson [33] concluded that the largest TECP values were found when the line of sight made a small angle with the phase front. The satellite trajectory of GPS 25 was almost parallel to the propagation direction of the phase front.

Figure 5 shows hourly histograms of the statistics of the phase front angle, $\alpha(t)$, and the horizontal velocity, $V_{h}(t)$, comprising all GPS satellites that passed near Huancayo between 21 and 24 UT. A total of 6 GPS satellites observed significant TEC perturbations associated with the TID. Each frame of Figure 5 contains a vertical arrow to point out the location of the statistical average. The propagation angle of the TIDs (left frames) indicates a consistent northward direction. The phase velocity of the TIDs varies between 60 and $120 \mathrm{~m} / \mathrm{s}$ with the maximum value occurring between 22 and 23 UT.

\section{Analysis of TID Characteristics Using Cross-Correlation Algorithm}

This section describes the cross-correlation method (CCM) and presents our results of the TID phase velocity, its direction of propagation and scale size. The aim of the CCM 
PRN 13

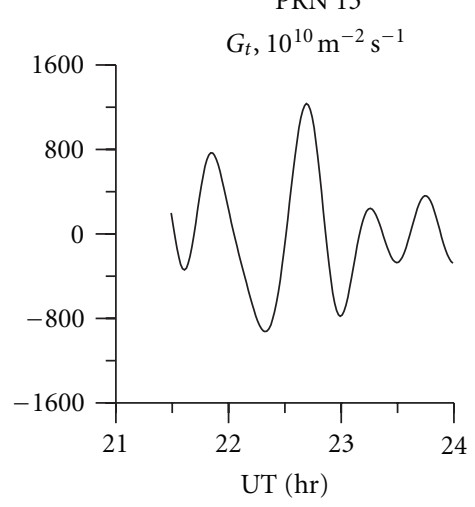

(a)

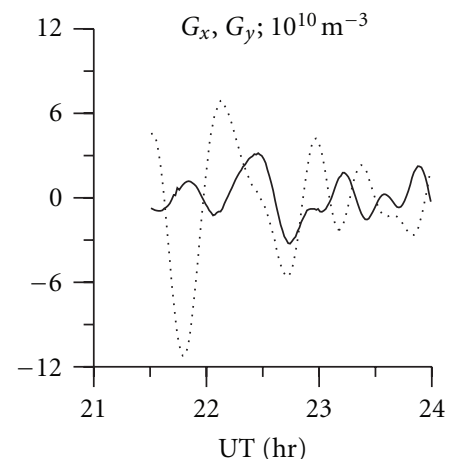

(b)

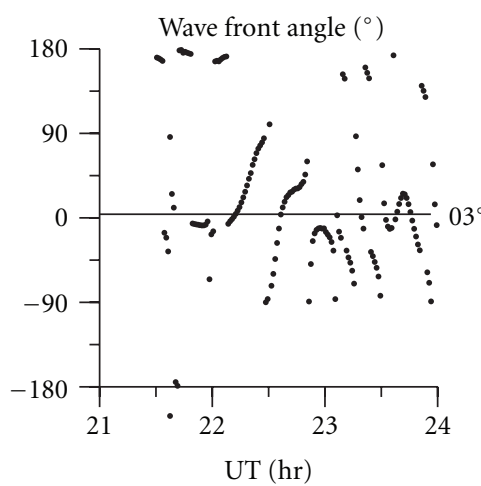

(c)

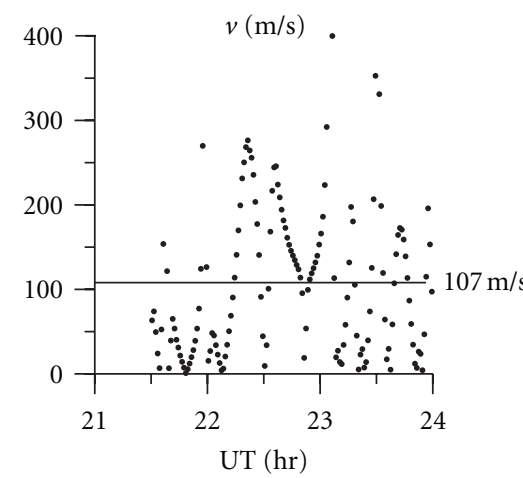

(d)

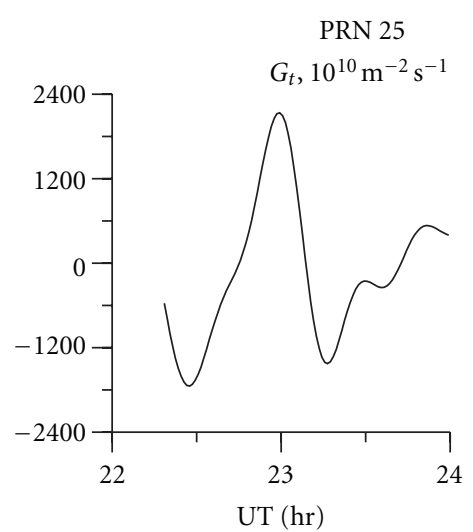

(e)

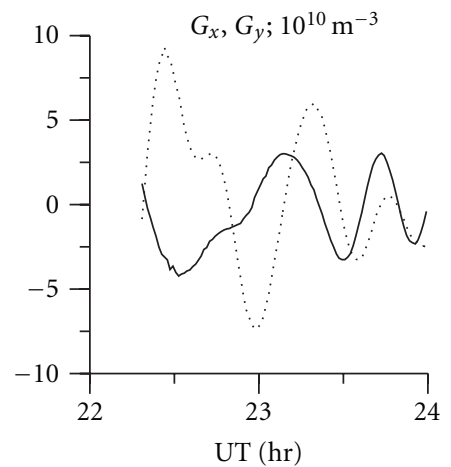

(f)

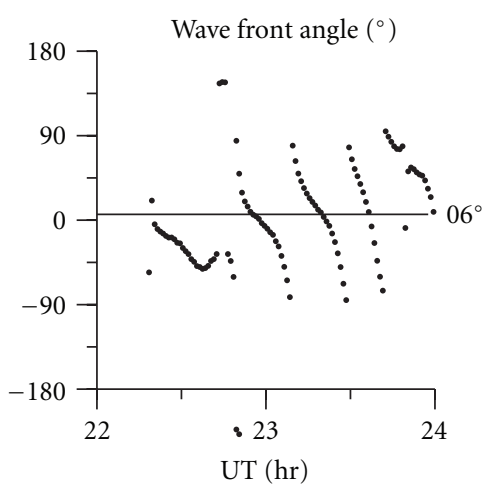

$(\mathrm{g})$

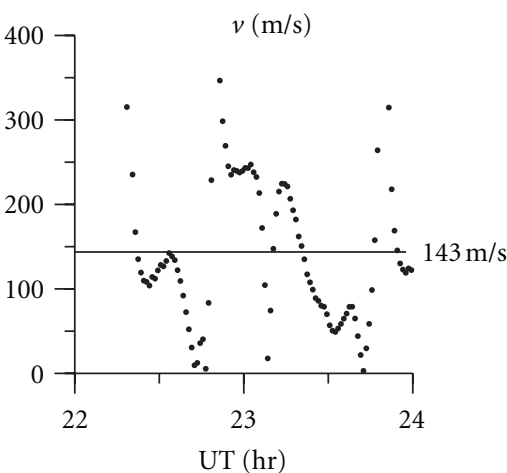

(h)

FIGURE 4: Derivation of different parameters used by the SADM-GPS method for GPS satellites 13 and 25. Panels (a) and (e) show $G_{t}$; (b) and (f) $G_{x}$ solid curve and $G_{x}$, dots; panels (c) and (g) show the direction of propagation $\alpha(t)$; (d) and (h) present the horizontal velocity, $V_{h}(t)$. 

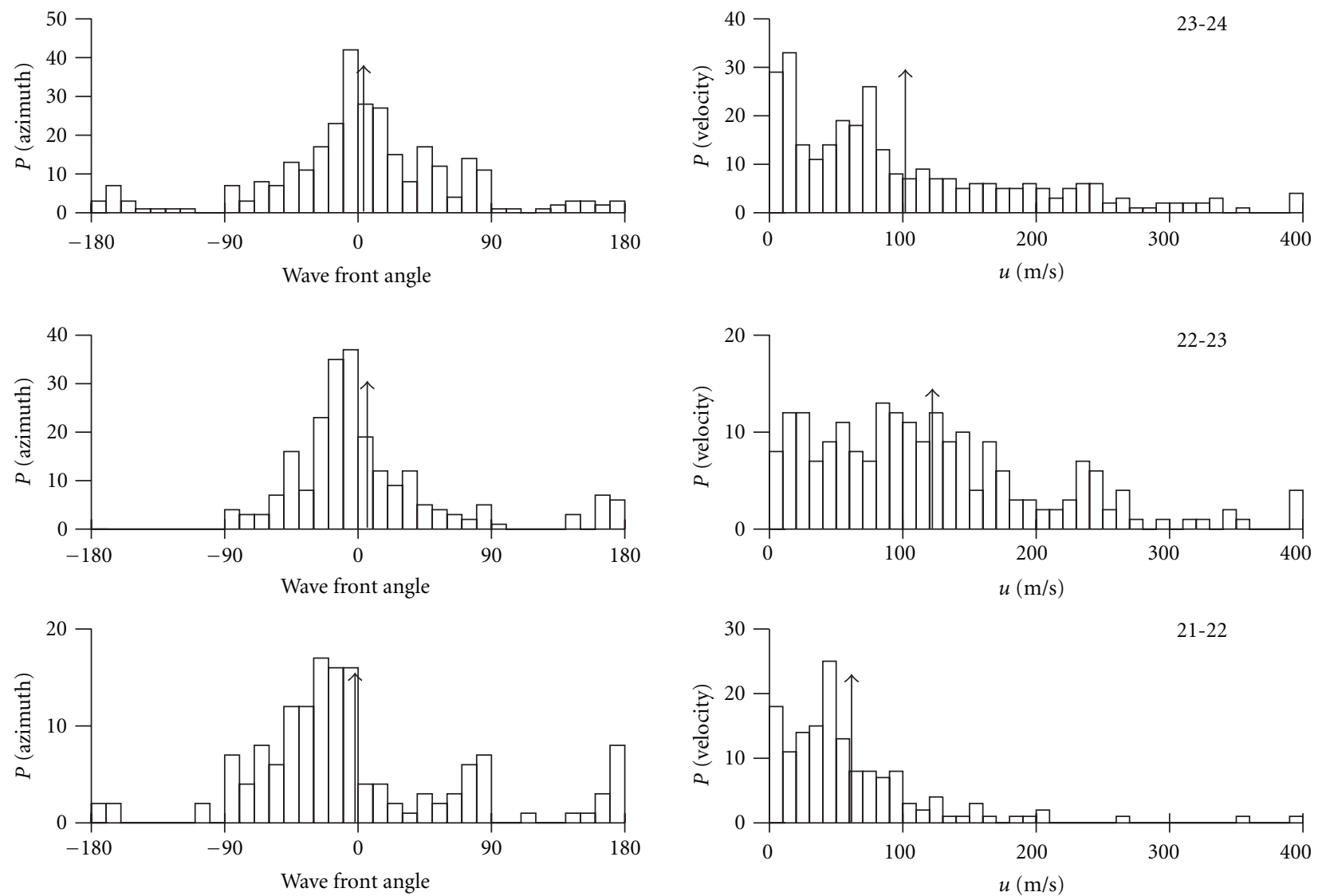

Figure 5: Distributions of the azimuth angle on the left and velocities on the right, corresponding to the calculated dependencies of $\alpha(t)$ and $V_{h}(t)$. The large arrows indicate the location of the mean value for each histogram.

method is to calculate the running cross-correlation function using TECP traces originated from any of the GPS satellites, but measured by two different receivers. The cross-correlation function is calculated every minute using segments of TECP data one hour long. We use TECP values from the same GPS satellite due to the motion of the satellite that produces the same temporal delay or advance on receivers placed at relatively close distances. This satellite motion effect can be removed after we calculate the phase velocity. If signals from different GPS satellites are used, it would be necessary to remove the effect of the satellite motion before the CCM method is applied. Figure 6 shows the results of the CCM method based on signals from the GPS 13 satellite recorded at Huancayo and Chupaca. The right frame of Figure 6 shows the same analysis using the TECP values measured at Huancayo and Sicaya. The lower panels show the TECP traces for GPS 13 in TEC units observed on July 20, 2008 and between 20 and 24 UT. The lower left frame displays the TECP values measured at Huancayo (blue line) and Chupaca (green line). Due to the coarse resolution of the plot, the small time delay between the traces becomes invisible. The cross-correlation functions, displayed in the upper frames, indicate that the time delay is of order $10 \mathrm{sec}$. Note the color scale of the crosscorrelation functions varies between 0.9 and 1.0 due to the high degree of coherence of both signals. To obtain the scale size of the TECP, we fitted an ensemble of monochromatic sinusoids to each segment one-hour long. The numbers printed in the lower frame point out the scale size in minutes of the best fit for each hour of TECP data. These results are affected by the motion of the GPS satellites and need to be corrected by subtracting the apparent motion of the subionospheric intersection in the direction perpendicular to the phase front. The propagation direction and velocity of the TID was obtained using:

$$
\begin{aligned}
\alpha(t)= & \arctan \left(\frac{Y_{C} T_{B-A}-Y_{A} T_{B-C}}{X_{C} T_{B-A}-X_{A} T_{B-C}}\right), \\
V_{h}(t)= & \frac{\left(Y_{C} \cos (\alpha(t))-X_{C} \sin (\alpha(t))\right)}{T_{B-C}} \\
& +w_{x}(t) \sin \alpha(t)+w_{y}(t) \cos \alpha(t) .
\end{aligned}
$$

$T_{B-A}$ and $T_{B-C}$ are the time delay between the Huancayo and Sicaya TEC perturbations and the offset time between the Huancayo and Chupaca TECP traces. As in the previous section, $w_{x}(t)$ and $w_{y}(t)$ are the projections of the sub-ionospheric intersection point velocity. A negative offset, as seen on the upper left frame indicates that the TECP observed at Huancayo lags Chupaca's TECP. A positive delay, as seen in the upper right frame is an indication that Huancayo's 

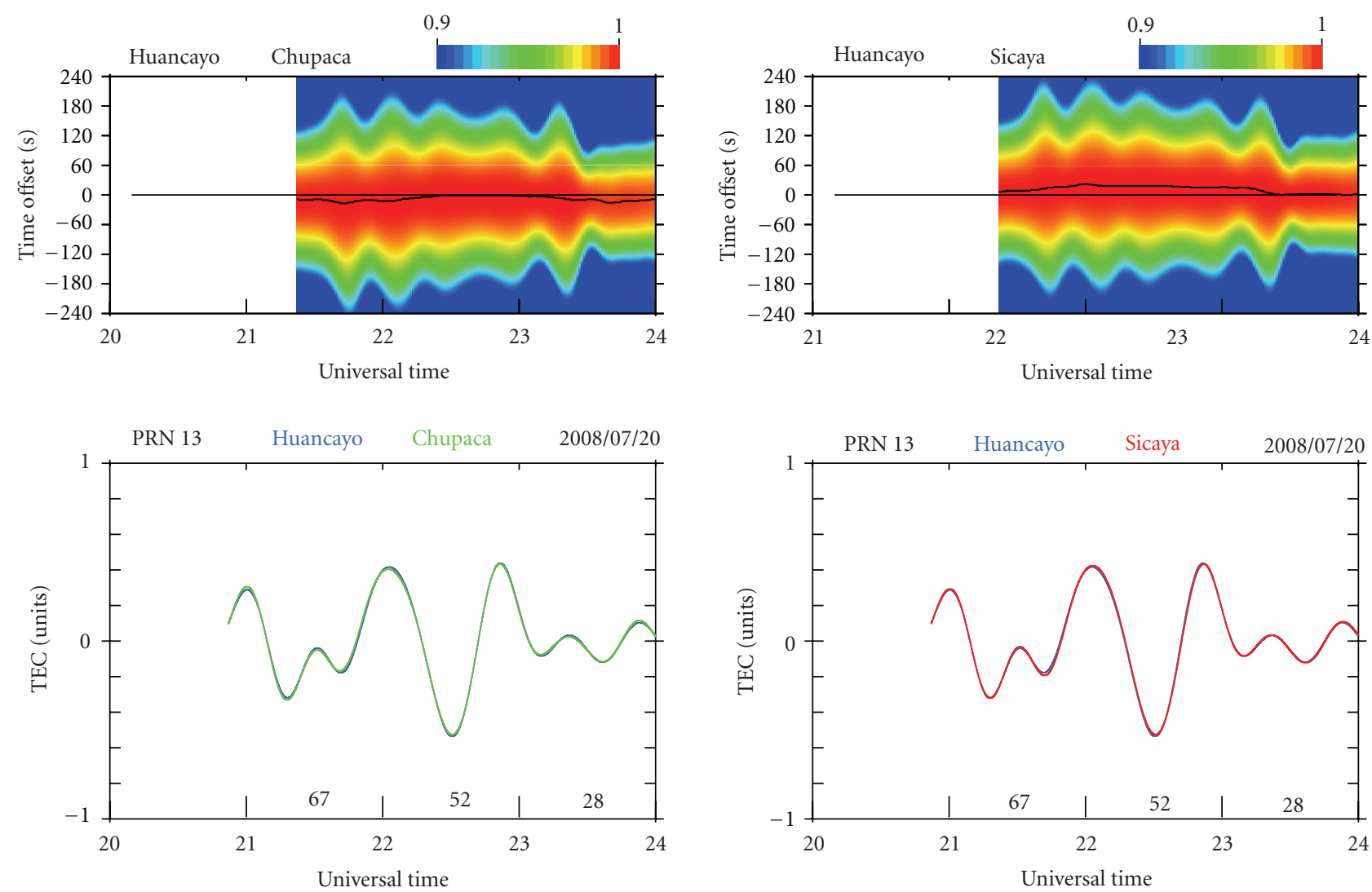

FIGURE 6: Cross-correlation functions (top panels) and TECP curves (lower frames) for GPS satellite 13. The left frames show the CCFs for TECP signals from Huancayo and Chupaca. The right panels correspond to Huancayo and Sicaya. The cross-correlation functions are plotted using a color scale that varies between 0.9 and 1.0. The black line that crosses near the middle corresponds to the maximum and it is taken as the time delay $\left(T_{B-A}\right.$ or $\left.T_{B-C}\right)$.

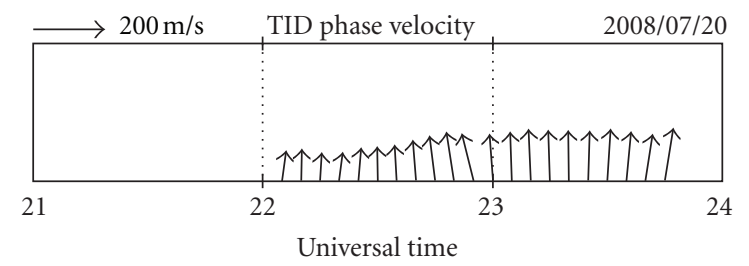

Figure 7: Phase velocity of the TIDs calculated using the CCM method and based on the data collected by the small array that operated near Huancayo.

TECP leads Sicaya's. Based on this timing relationship, it can be concluded that the perturbation associated with the TID was seen first at Chupaca, located further south, then at Huancayo placed near the center of the array, and later at Sicaya placed further north (Figure 1). This sequence of events indicates that the TID motion was in the northward direction.

A more quantitative estimation of the TID phase velocity was obtained using (8) and (9). Figure 7 shows the magnitude and direction of the TID phase velocity represented by arrows. The size of the arrow is proportional to the phase velocity and the azimuthal rotation of the vector is a function of the propagation angle. An arrow pointing to the top indicates a propagation direction toward geographic north. It is evident that between 22 and 24 UT, the TID moved toward geographic north. A wavelength equal to $449 \mathrm{~km}$ is obtained using the true phase velocity of the TID and the velocity of the satellite sub-ionospheric intersection point. The phase velocity varied between $84 \mathrm{~m} / \mathrm{s}$ and $144 \mathrm{~m} / \mathrm{s}$ near 24 UT. These values are consistent with the results of the SADMGPS method (Section 3).

\section{Analysis of the TID Characteristics Using GPS Receivers Spaced Hundreds of Kilometers}

We also report our investigation of the TID morphological properties using GPS receivers that belong to the low-latitude ionospheric sensor network (LISN) that operate at different cities separated by hundreds of kms. LISN is a distributed observatory designed to nowcast the state and dynamics of the low-latitude ionosphere over South America. The LISN observatory is comprised of GPS receivers, flux-gate magnetometers and vertical incidence-pulsed ionospheric radar (VIPIR) ionosondes. A unique feature of the observatory is the ability of all the instruments to relay their observables in a near real-time manner via Internet. At the time of this 

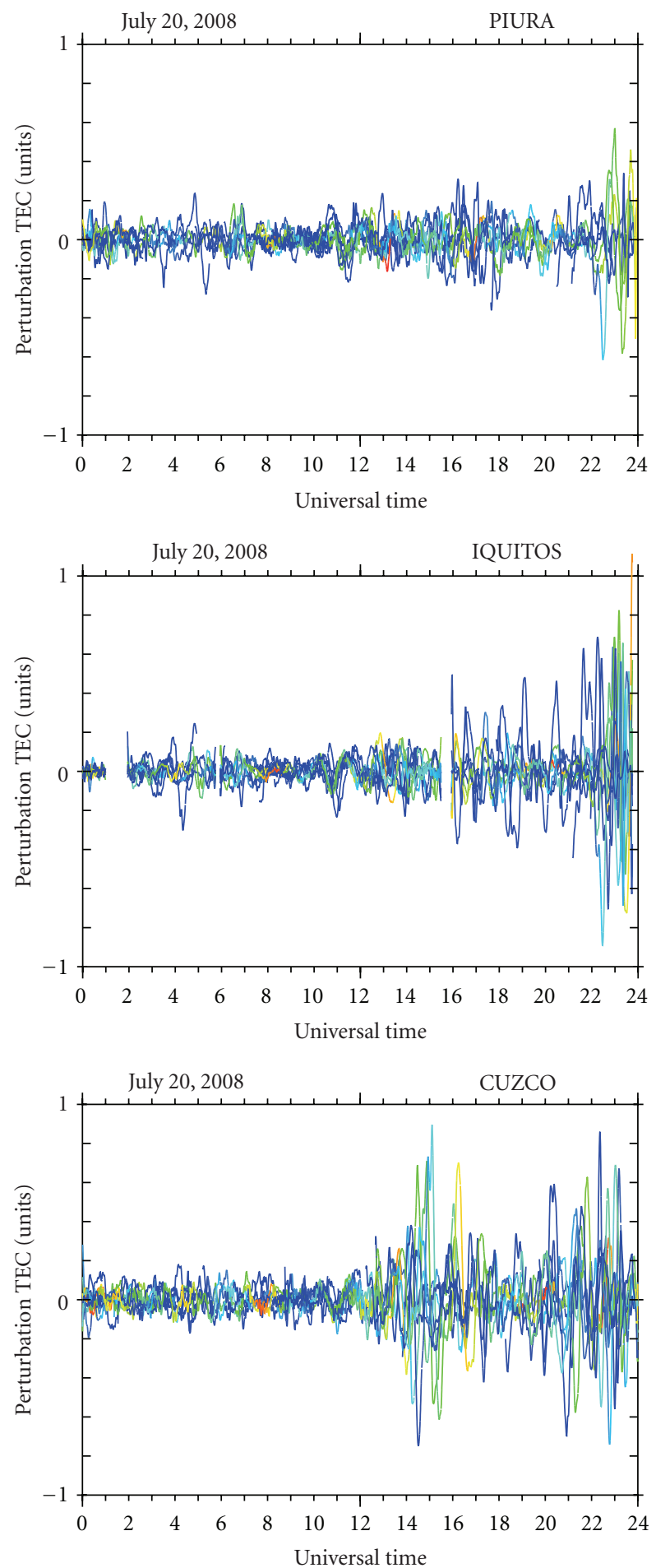

FIGURE 8: Same as Figure 3, but for three of the LISN stations. From top to bottom: Piura, Iquitos, and Cuzco. These TECP values were obtained on July 20, 2008.

paper (November 2011), 44 dual-frequency GPS receivers were part of the LISN network. During the time of the campaign a total of 126 GPS receivers-including the LISN GPS receivers-operated in the South American continent. TEC perturbations from these receivers, especially the ones located close to Huancayo, were used to provide supportive evidence for the TID dynamic characteristics and give a regional view of the presence of TIDs over South America.

Figure 8 shows the TECP values measured on July 20, 2008 at Piura $\left(5.17^{\circ} \mathrm{S} ; 80.63^{\circ} \mathrm{W}\right)$, Iquitos $\left(3.77^{\circ} \mathrm{S} ; 73.27^{\circ} \mathrm{W}\right)$ and Cuzco $\left(13.52^{\circ} \mathrm{S}, 71.96^{\circ} \mathrm{W}\right)$. The TEC perturbations in Piura were seen mainly between 21 and 24 UT. Iquitos, 

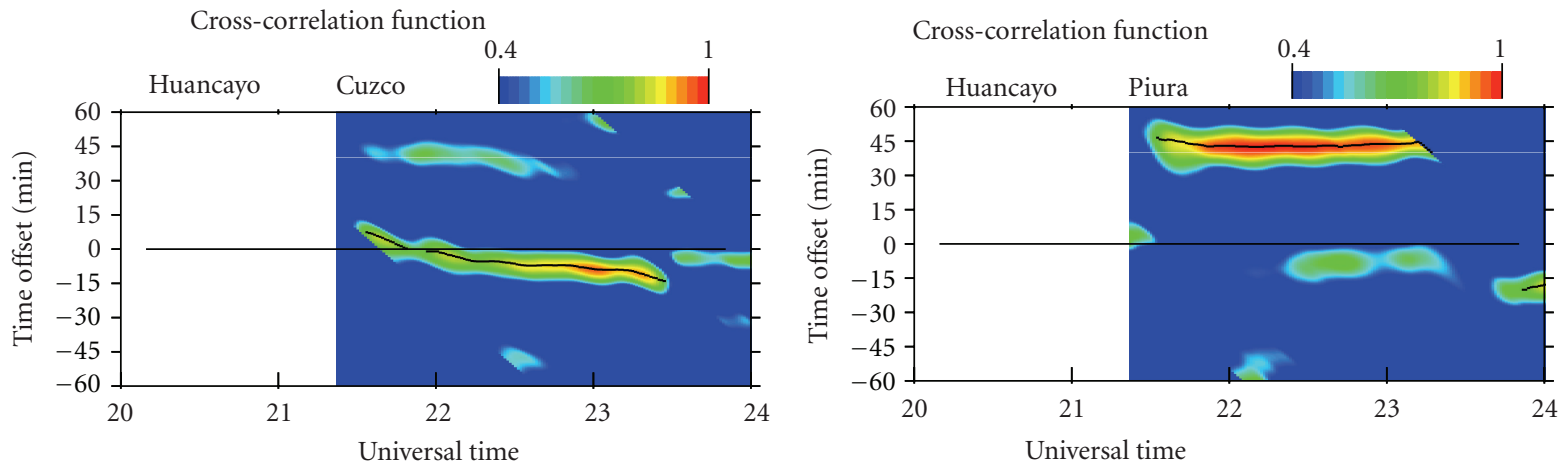

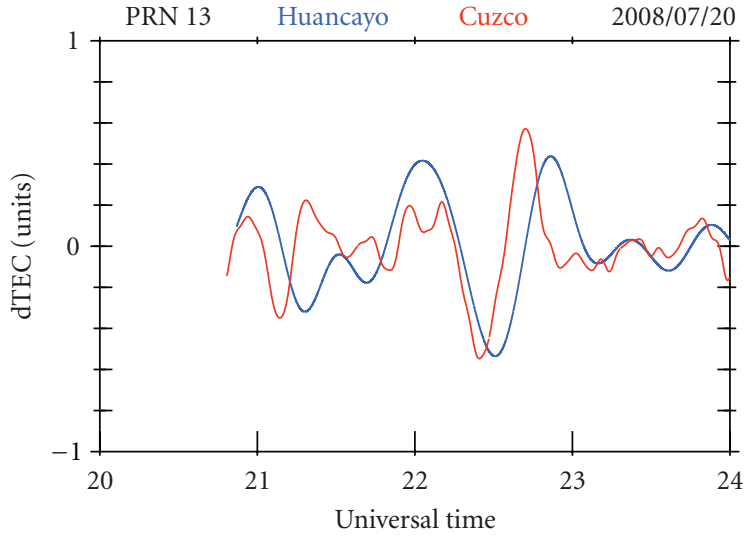

(a)

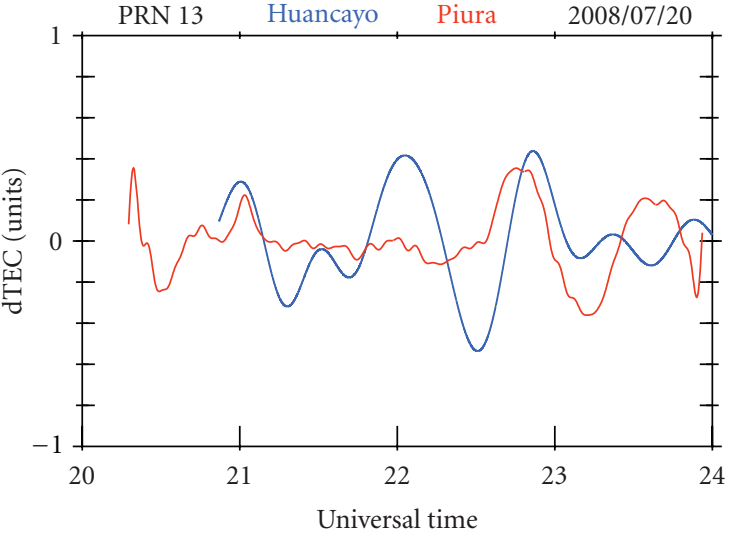

(b)

Figure 9: Same as Figure 6, but for CCF between Huancayo and Cuzco on the left side and Huancayo and Piura on the right side.

Huancayo; Cuzco; Piura

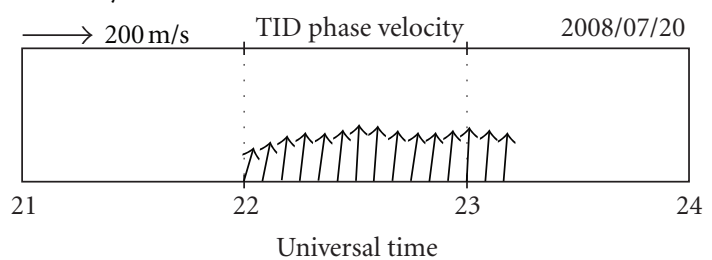

FIGURE 10: Same as Figure 7, but for TID's phase velocities that were calculated using TECP values from Huancayo, Cuzco, and Piura.

located in the north-eastern side of Peru, registered large TECP values between 16 and 24 UT. Cuzco, located close to the magnetic equator and $250 \mathrm{~km}$ east of Huancayo, observed large TEC values between 14 and 24 UT. These 3 stations show TECP values much larger than the noise level almost simultaneously. However, the TECP amplitude is different at different stations. Figure 8 suggests that between 21 and 24 UT, TIDs existed in the western region of South America. Below, we discuss further this finding.

The lower panels of Figure 9 display the TECP values for Huancayo (in blue), Cuzco (left panel in red), and Piura (right panel in red). The upper left frame presents the CCFs calculated using the Huancayo and Cuzco TECPs, showing a relatively short delay that varies between +10 and -15 min.
Note that due to the large distances between the stations, the time delays between these station's TECP values are of order of tens of minutes. The right upper frame of Figure 9 displays CCFs for Huancayo and Piura. The time delay between the TECP values for these two stations is $\sim 45 \mathrm{~min}$. This value agrees quite well with the time offset that is observed in the lower panel. Visual inspection of the minimum value of the blue curve (Huancayo) observed at $\sim 2230$ UT and the minimum value of the red trace seen at $\sim 2315$ UT indicates that the Huancayo TECP values lead the Piura TECP values by $45 \mathrm{~min}$. Note that CCFs in the upper panels also show secondary maximum with much smaller amplitudes $(\sim 0.6)$ and spaced roughly $50-55 \mathrm{~min}$ away from the primary maximum. In the case of a perfect sinusoidal oscillation, this spacing would be equal to the wavelength of the sinusoid. However, since MS-TIDs are more properly viewed as traveling wave packets [30], this spacing corresponds to the scale size of the TID packet. It is also pointed out that the CCF amplitude scale was limited to values above 0.4 and did not include negative values as they would indicate anticorrelations. If we had used a CCF amplitude scale varying between -1 and 1 , a negative peak would have been observed between the primary and secondary maxima.

Figure 10 shows the TIDs phase velocity that was obtained using (8) and (9) and the time delays presented in Figure 9. The similarity of the angle $\alpha(t)$ that was derived based on measurements by GPS receivers separated by 


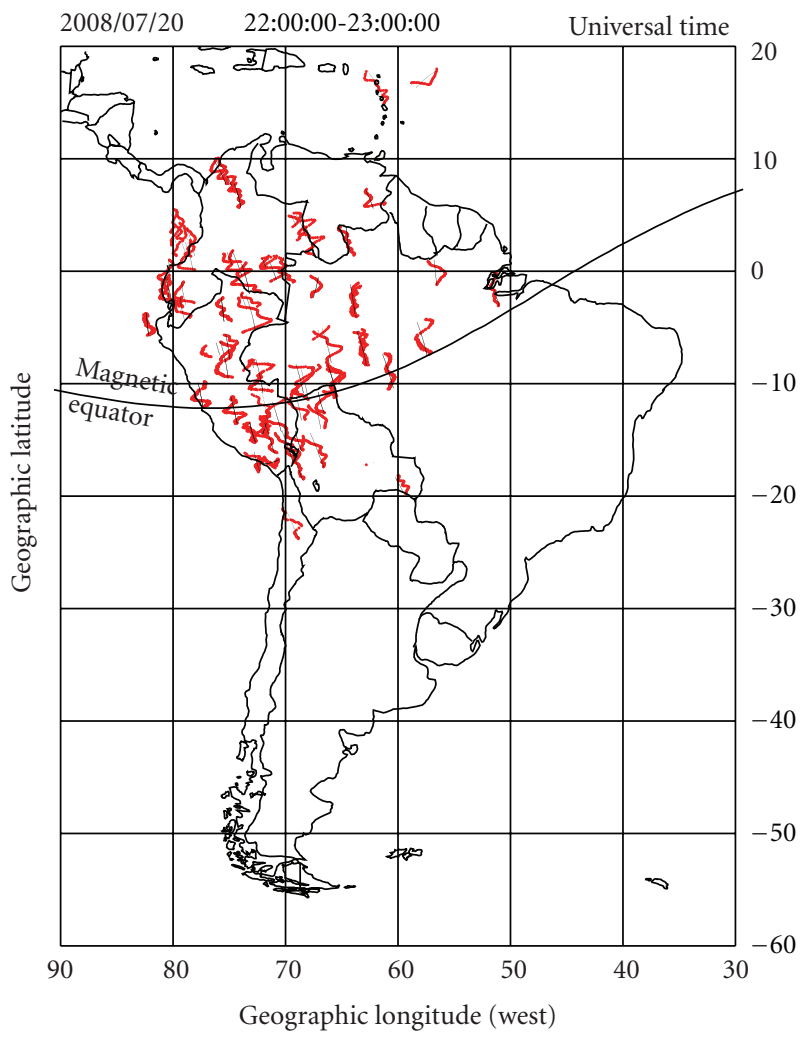

FIGURE 11: TEC perturbations recorded by several GPS sites that operated in South America on July 20, 2008. The red traces correspond to the TECP values after subtracting the daily variability.

hundreds of kms and the $\alpha(t)$ calculations based on GPS receivers that were installed only few $\mathrm{kms}$ apart is striking. Figures 7 and 10 show $\alpha(t)$ to be directed very closely in the geographic northward direction. However, the amplitude of the horizontal phase velocity $V_{h}(t)$, seen at $2230 \mathrm{UT}$, varies between $100 \mathrm{~m} / \mathrm{s}$ in Figure 7 and $160 \mathrm{~m} / \mathrm{s}$ in Figure 10. The difference in the $V_{h}(t)$ values is reduced at $2300 \mathrm{UT}$, when $135 \mathrm{~m} / \mathrm{s}$ is shown in Figure 7 and $147 \mathrm{~m} / \mathrm{s}$ in Figure 10.

The TEC perturbations for all 126 GPS receivers that operated in South America on July 20, 2008 are presented in Figure 11. These GPS receivers provide a good coverage over the whole South American continent, except for latitudes southward of $40^{\circ} \mathrm{S}$. Figure 11 shows TECP values in red, centered along the satellite trajectory (thin black line), that have amplitudes higher than 0.4 TEC units. This baseline value is about 4 times the noise level of TEC fluctuations. The TECP traces of Figure 11 demonstrate that TIDs were restricted to the north-western side of South America between 2200 and 2300 UT. Similar Figures corresponding to earlier times, not shown here, display the TECP traces to be located further south. They indicate that the TIDs were propagating northward and were probably originated in the northern part of Chile (lat. $\left.=20^{\circ} \mathrm{S}\right)$.

\section{Discussion}

We conducted a campaign at Huancayo in Peru to assess the effectiveness of GPS receivers in detecting and measuring the morphology and characteristics of TIDs that circulate at low latitudes. To accomplish this, we temporarily installed two additional GPS receivers near the Huancayo station to form 3 closely spaced GPS receivers having a geometry that can be used as a GPS radio-interferometer. The TIDs that were observed during the campaign can be classified as mediumscale disturbances (MSTIDs). They have horizontal phase velocities between 100 and $250 \mathrm{~m} / \mathrm{s}$, wavelengths of several hundred kilometers and periods between 15 and $60 \mathrm{~min}$ [34]. We also indicate that the TIDs observed on July 20, 2008 are associated with AGW, that are likely generated in the lower atmosphere.

To derive the TID characteristics we applied two different methods (SADM-GPS and CCM) and used two different datasets. The SADM-GPS is a statistical method that provides the correct phase velocity and the angle of propagation of the TIDs. However, it requires averaging the derived parameters for one hour and for all the GPS satellites with look angles above $35^{\circ}$ elevation. The CCM method seems more robust, and it is less affected by the fact that more than one AGW may be circulating across the volume probed by the GPS receivers. A more detailed comparison of the results provided by the SADM-GPS and the CCM methods shows an excellent agreement in the angle of propagation, as both indicate the angle of propagation to be equal to $+3^{\circ} \pm 5^{\circ}$. Calculation of the phase velocity with the SADM-GPS method indicates an average velocity of $120 \mathrm{~m} / \mathrm{s}$ between 22 and 23 UT. The CCM method is more precise and indicates that 
the phase velocity varies between 84 and $130 \mathrm{~m} / \mathrm{s}$ during the same interval.

We have also applied the CCM method to TECP values collected by other GPS receivers that belong to the LISN network and are operating at Piura, Cuzco, and Iquitos, all of them in Peru. We found very good correlation, in excess of 0.9 , between the TECP traces corresponding to stations placed hundreds of kms away. We were able to calculate that the angle of propagation $\alpha(t)$ was close to $0^{\circ}$ (northward propagation) and the horizontal phase velocity $V_{h}(t)$ was between 130 and $160 \mathrm{~m} / \mathrm{s}$. The latter values are larger than the numbers obtained using the small interferometer array of 3 GPS receivers. However, several geometrical considerations need to be accounted with more precision. One of them is the fact that the projection of the apparent satellite motion over the propagation direction of the TID may vary during the $45 \mathrm{~min}$ travel time between the LISN stations. It is also possible that the TID may not be a plane wave, and the wave front may have a circular shape. Figure 11 shows that the region populated by TIDs seems to extend over a large region. TECP images obtained prior to Figure 11 indicate that the TIDs originated near latitude $=20^{\circ} \mathrm{S}$. We do not know if they were produced by deep convection cells or were a response to orographic features in northern Chile. We believe that the success in getting good agreements is partially due to the scale size of the TIDs that was over $400 \mathrm{kms}$. This distance is larger than the separation between GPS sites. Further studies will determine the preferred scale sizes, the phase velocity, and the direction of propagation of the TIDs using all the GPS receivers that are operating in South America.

The GPS radio-interferometry (GPS-RI) method is one of the most effective techniques with which to observe GW/ TID activity at low latitudes. TIDs are notoriously difficult to characterize due to their large size. However, GPS-RI enables characterization of their large-scale features with ease, including their horizontal wavelength, phase speed, and propagation azimuths. Since GPS-RI is based on radio-frequency measurements, the technique is not limited by weather considerations; data may be taken at all times, unlike with optical observations. GPS receivers are much less expensive to deploy than other instruments of similar or lesser sensitivity to TIDs, including phased-array radars, steerablebeam radar systems, and ionosondes. Moreover, an extensive global array of GPS receivers already exists, allowing for widespread, continuous monitoring of TID activity. Never before has this been possible.

Additional observations, when combined with GPS-RI observations, yield an even more complete TID characterization. In particular, measurements of the thermospheric neutral wind may be used to calculate TID intrinsic frequency by the relation: $\omega=K_{H}\left(V_{h}(t),-U_{n}\right)$, where $\omega$ is the intrinsic frequency, $K_{H}$ is the TID horizontal wavenumber of the TID, and $U_{n}$ is the neutral mean wind in the direction of TID propagation. Further, if the intrinsic frequency is known, TID vertical wavelength may be calculated by using one of the dispersion relations $[35,36]$. Thus, a very complete picture of TIDs results with only GPS-RI and thermospheric wind observations: TID horizontal wavelength, vertical wavelength, intrinsic frequency, amplitude, and propagation direction are all specified. When GPS-RI receivers are colocated with other instrumentation, observed and calculated parameters may be cross-validated. Most importantly, TID horizontal wavelengths and propagation azimuths may be compared to GPS-RI results when favorable seeing conditions pertain, providing a crucial validation of this technique. Calculated values of intrinsic frequency and vertical wavelength may be compared with ionosonde-derived values.

Low-latitude TIDs are important to study because they are a key mechanism by which energy and momentum are deposited into the thermosphere. Observation of TIDs at altitudes near where they break would enable a direct investigation of this process for the first time. Additionally, TIDs are thought to be a key seed mechanism of plasma depletions. While evidence of TID seeding was found during the SpreadFEx campaigns [37], never before has it been possible to continuously monitor TID activity on the F-region bottomside during the crucial early-evening hours, when seeding occurs. An investigation of the presence/absence of TID activity on the bottomside will help determine the importance of TIDs relative to other mechanisms toward seeding equatorial plasma depletions.

Characterization of TID large-scale features should help establish which types of TIDs are most effective seeds. For example, is a minimum perturbation amplitude needed to seed depletions? TID wavelength and propagation azimuth, also characterized by GPS-RI arrays, are also thought to influence seeding effectiveness. Observations [38] suggest that TIDs must have a minimum horizontal wavelength to effectively seed depletions. Model results and seeding theory indicate that a TID must propagate nearly parallel to the geomagnetic equator to be maximally effective at seeding plasma depletions. GPS-RI makes it possible to directly investigate the impact of the variation of horizontal wavelength and propagation azimuth on plasma depletion formation.

\section{Conclusions}

We have presented calculations of the phase velocity, scale size, and angle of propagation of TIDs that are likely originated by gravity waves that are propagating in the northwestern side of the South American continent. The values that were obtained using the SADM-GPS and the CCM methods are consistent with values of medium-scale TIDs observed by other authors. We calculated that on July 20, 2008, the propagation velocity and the azimuth direction of the TIDs were $140 \mathrm{~m} / \mathrm{s}$ and parallel to geographic north.

We also applied the CCM method to stations separated by hundreds of $\mathrm{km}$, like Huancayo, Cuzco, and Piura. It was unexpected to find a large coherence between the TECP traces from all the adjacent stations, allowing us to determine the morphological characteristics of the TIDs. This result opens the possibility of using measurements of the TEC perturbations by the GPS receivers already deployed in South America to monitor TID activity. We have conducted two more campaigns using a small network of three receivers that were placed near a new ionosonde that operated at the Jicamarca radar site between 2009 and 2010. We expect to publish these results in the near future. 


\section{Acknowledgments}

The authors would like to thank International GNSS service (IGS), Geocentric Reference System for the Americas (SIRGAS), and Dr. Michael Bevis from Ohio State UniversityCentral and Southern Andes GPS Project (OSU-CAP) for providing GPS data. One of the authors, C. E. Valladares, was partially supported by Air Force Research Laboratory contract FA8718-09-C-0041, NSF Grant ATM-0521487, and NASA LWS Grant NNX11AP02G. The Low-Latitude Ionospheric Sensor Network (LISN) is a project led by Boston College in collaboration with the Geophysical Institute of Peru, and other institutions that provide information in benefit of the scientific community. They thank all organizations and persons that are supporting and operating receivers in LISN. They thank Robert Sheehan for his helpful comments and suggestions on the paper.

\section{References}

[1] W. H. Hooke, "Ionospheric irregularities produced by internal atmospheric gravity waves," Journal of Atmospheric and Terrestrial Physics, vol. 30, no. 5, pp. 795-823, 1968.

[2] T. M. Georges and W. H. Hooke, "Wave- induced fluctuations in ionospheric electron content. A model indicating some observational biases," Journal of Geophysical Research, vol. 75, no. 31, pp. 6295-6308, 1970.

[3] M. J. Davis, "The integrated ionospheric response to internal atmospheric gravity waves," Journal of Atmospheric and Terrestrial Physics, vol. 35, no. 5, pp. 929-959, 1973.

[4] M. C. Kelley and S. Fukao, "Turbulent upwelling of the midlatitude ionosphere. II-theoretical framework," Journal of Geophysical Research, vol. 96, pp. 3747-3753, 1991.

[5] T. L. Beach, "Total electron content variations due to nonclassical traveling ionospheric disturbances: theory and Global Positioning System observations," Journal of Geophysical Research A, vol. 102, no. 4, pp. 7279-7292, 1997.

[6] C. A. Miller, "Electrodynamics of midlatitude spread F 1. Observations of unstable, gravity wave-induced ionospheric electric fields at tropical latitudes," Journal of Geophysical Research A, vol. 102, no. 6, pp. 11521-11532, 1997.

[7] A. R. Jacobson, R. Carlos, R. Massey, and G. Wu, "Observations of traveling ionospheric disturbances with a satellitebeacon radio interferometer: seasonal and local time behavior," Journal of Geophysical Research, vol. 100, pp. 1653-1665, 1995.

[8] P. J. Sultan, "Linear theory and modeling of the RayleighTaylor instability leading to occurrence of equatorial spread F," Journal of Geophysical Research, vol. 101, pp. 26819-26827, 1996.

[9] R. F. Woodman and C. La Hoz, "Radar observations of F region equatorial irregularities," Journal of Geophysical Research, vol. 81, no. 31, pp. 5447-5466, 1976.

[10] A. J. Scannapieco and S. L. Ossakow, "Nonlinear equatorial spread F evolution," Geophysical Research Letters, vol. 3, pp. 451-454, 1976.

[11] M. C. Kelley, M. F. Larsen, C. LaHoz, and J. P. McClure, "Gravity wave initiation of equatorial spread F: a case study," Journal of Geophysical Research, vol. 86, pp. 9087-9100, 1981.

[12] E. Kudeki, A. Akgiray, M. Milla, J. L. Chau, and D. L. Hysell, "Equatorial spread-F initiation: post-sunset vortex, thermospheric winds, gravity waves," Journal of Atmospheric and
Solar-Terrestrial Physics, vol. 69, no. 17-18, pp. 2416-2427, 2007.

[13] R. T. Tsunoda, "Seeding of equatorial plasma bubbles with electric fields from an $E_{s}$-layer instability," Journal of Geophysical Research A, vol. 112, no. 6, Article ID A06304, 13 pages, 2007.

[14] S. Fukao, M. Kelley, and T. Shirakawa, "Turbulent upwelling of the mid-latitude ionosphere. I- Observational results by the MU radar," Journal of Geophysical Research, vol. 96, pp. 37253746, 1991.

[15] W. L. Oliver, S. Fukao, M. Sato, Y. Otsuka, T. Takami, and T. Tsuda, "Middle and upper atmosphere radar observations of the dispersion relation for ionospheric gravity waves," Journal of Geophysical Research, vol. 100, no. 12, pp. 23763-23768, 1995.

[16] M. K. Ejiri, K. Shiokawa, T. Ogawa, K. Igarashi, T. Nakamura, and T. Tsuda, "Statistical study of short-period gravity waves on $\mathrm{OH}$ and $\mathrm{OI}$ nightglow images at two separated sites," Journal of Geophysical Research D, vol. 108, no. 21, pp. 1-12, 2003.

[17] S. Suzuki, K. Shiokawa, Y. Otsuka, T. Ogawa, and P. Wilkinson, "Statistical characteristics of gravity waves observed by an all-sky imager at Darwin, Australia," Journal of Geophysical Research D, vol. 109, no. 20, pp. D20-DS07, 2004.

[18] A. A. Pimenta, M. C. Kelley, Y. Sahai, J. A. Bittencourt, and P. R. Fagundes, "Thermospheric dark band structures observed in all-sky OI $630 \mathrm{~nm}$ emission images over the Brazilian lowlatitude sector," Journal of Geophysical Research A, vol. 113, no. 1, Article ID A01307, 9 pages, 2008.

[19] D. C. M. Amorim, A. A. Pimenta, J. A. Bittencourt, and P. R. Fagundes, "Long-term study of medium-scale traveling ionospheric disturbances using O i $630 \mathrm{~nm}$ all-sky imaging and ionosonde over Brazilian low latitudes," Journal of Geophysical Research A, vol. 116, no. 6, Article ID A06312, 7 pages, 2011.

[20] K. Shiokawa, C. Ihara, Y. Otsuka, and T. Ogawa, "Statistical study of nighttime medium-scale traveling ionospheric disturbances using midlatitude airglow images," Journal of Geophysical Research A, vol. 108, no. 1, article 1052, 2003.

[21] J. L. Bougeret, "Some effects produced by the ionosphere on radio interferometry-fluctuations in apparent source position and image distortion," Astronomy \& Astrophysics, vol. 96, pp. 259-266, 1981.

[22] C. Mercier, "Observations of atmospheric gravity waves by radiointerferometry," Journal of Atmospheric and Terrestrial Physics, vol. 48, no. 7, pp. 605-624, 1986.

[23] A. R. Jacobson and W. C. Erickson, "A method for characterizing transient ionospheric disturbances using a large radio telescope array," Astronomy \& Astrophysics, vol. 257, pp. 401409, 1992.

[24] C. E. Valladares, J. Villalobos, M. A. Hei et al., "Simultaneous observation of traveling ionospheric disturbances in the Northern and Southern Hemispheres," Annales Geophysicae, vol. 27, no. 4, pp. 1501-1508, 2009.

[25] M. C. Kelley, The Earth's Ionosphere, Academic Press, London, UK, 1989.

[26] M. W. Kirkland and A. R. Jacobson, "Drift-parallax determination of the altitude of traveling ionospheric disturbances observed with the Los Alamos radio-beacon interferometer," Radio Science, vol. 33, no. 6, pp. 1807-1825, 1998.

[27] S. L. Vadas, "Horizontal and vertical propagation and dissipation of gravity waves in the thermosphere from lower atmospheric and thermospheric sources," Journal of Geophysical Research A, vol. 112, no. 6, Article ID A06305, 23 pages, 2007. 
[28] E. L. Afraimovich, K. S. Palamartchouk, and N. P. Perevalova, "GPS radio interferometry of travelling ionospheric disturbances," Journal of Atmospheric and Solar-Terrestrial Physics, vol. 60, no. 12, pp. 1205-1223, 1998.

[29] E. L. Afraimovich, E. A. Kosogorov, L. A. Leonovich, K. S. Palamartchouk, N. P. Perevalova, and O. M. Pirog, "Determining parameters of large-scale traveling ionospheric disturbances of auroral origin using GPS-arrays," Journal of Atmospheric and Solar-Terrestrial Physics, vol. 62, no. 7, pp. 553-565, 2000.

[30] E. L. Afraimovich, N. P. Perevalova, and S. V. Voyeikov, "Traveling wave packets of total electron content disturbances as deduced from global GPS network data," Journal of Atmospheric and Solar-Terrestrial Physics, vol. 65, no. 11-13, pp. 1245-1262, 2003.

[31] D. C. Fritts, M. A. Abdu, B. R. Batista et al., "Overview and summary of the Spread F Experiment (SpreadFEx)," Annales Geophysicae, vol. 27, pp. 2141-2155, 2009.

[32] E. Sardón and N. Zarraoa, "Estimation of total electron content using GPS data: how stable are the differential satellite and receiver instrumental biases?" Radio Science, vol. 32, no. 5, pp. 1899-1910, 1997.

[33] C. Mercier and A. R. Jacobson, "Observations of atmospheric gravity waves by radio interferometry: are results biased by the observational technique?" Annales Geophysicae, vol. 15, no. 4, pp. 430-442, 1997.

[34] K. Hocke and K. Schlegel, "A review of atmospheric gravity waves and travelling ionospheric disturbances: 1982-1995," Annales Geophysicae, vol. 14, no. 9, pp. 917-940, 1996.

[35] J. K. Hargreaves, The Solar-Terrestrial Environment: An Introduction to Geospace, Cambridge University Press, Cambridge, UK, 1992.

[36] E. A. Kherani, M. A. Abdu, E. R. D. De Paula, D. C. Fritts, J. H. A. Sobral, and F. C. de Meneses Jr., "The impact of gravity waves rising from convection in the lower atmosphere on the generation and nonlinear evolution of equatorial bubble," Annales Geophysicae, vol. 27, no. 4, pp. 1657-1668, 2009.

[37] D. C. Fritts, S. L. Vadas, D. M. Riggin et al., "Gravity wave and tidal influences on equatorial spread $\mathrm{F}$ based on observations during the Spread F Experiment (SpreadFEx)," Annales Geophysicae, vol. 26, no. 11, pp. 3235-3252, 2008.

[38] M. A. Hei, R. A. Heelis, and J. P. McClure, "Seasonal and longitudinal variation of large-scale topside equatorial plasma depletions," Journal of Geophysical Research, vol. 110, Article ID A12315, 13 pages, 2005. 

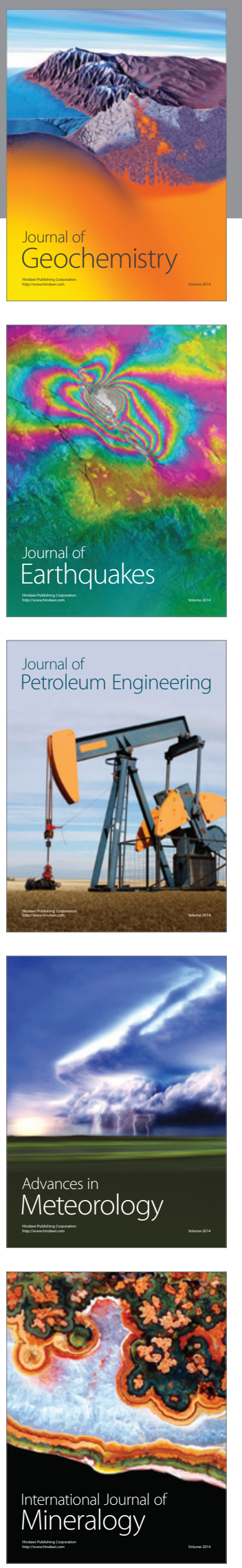
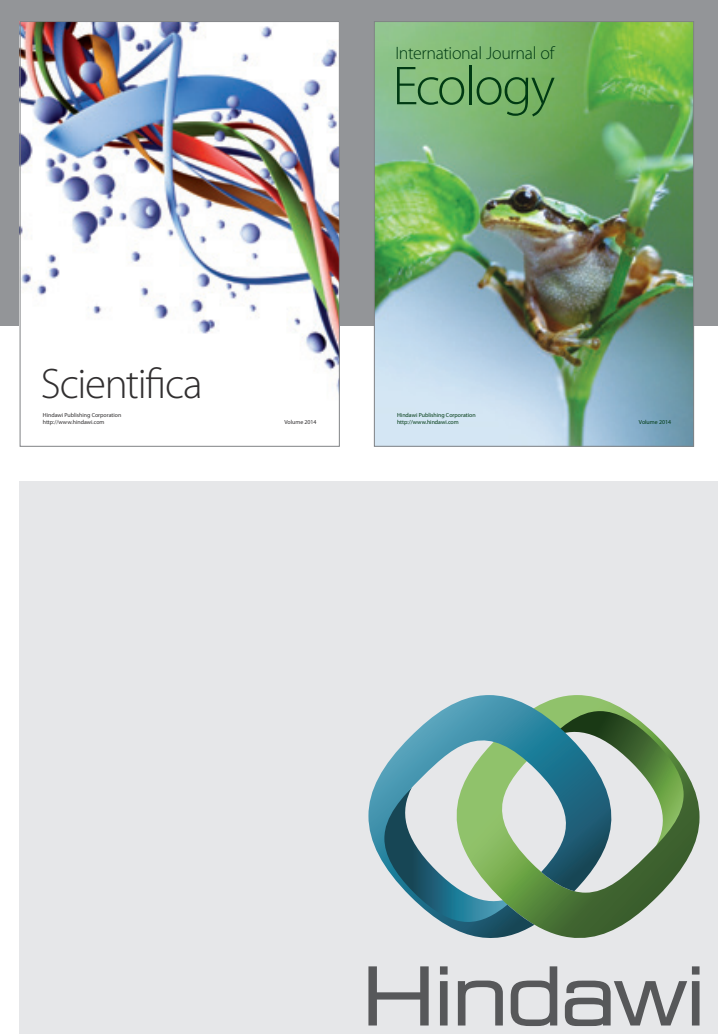

Submit your manuscripts at http://www.hindawi.com
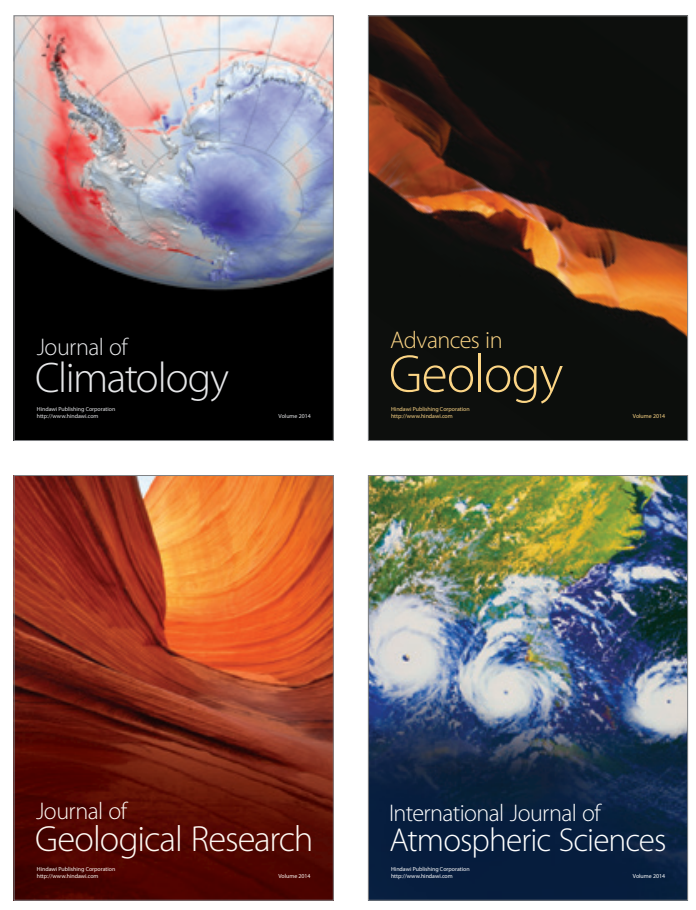
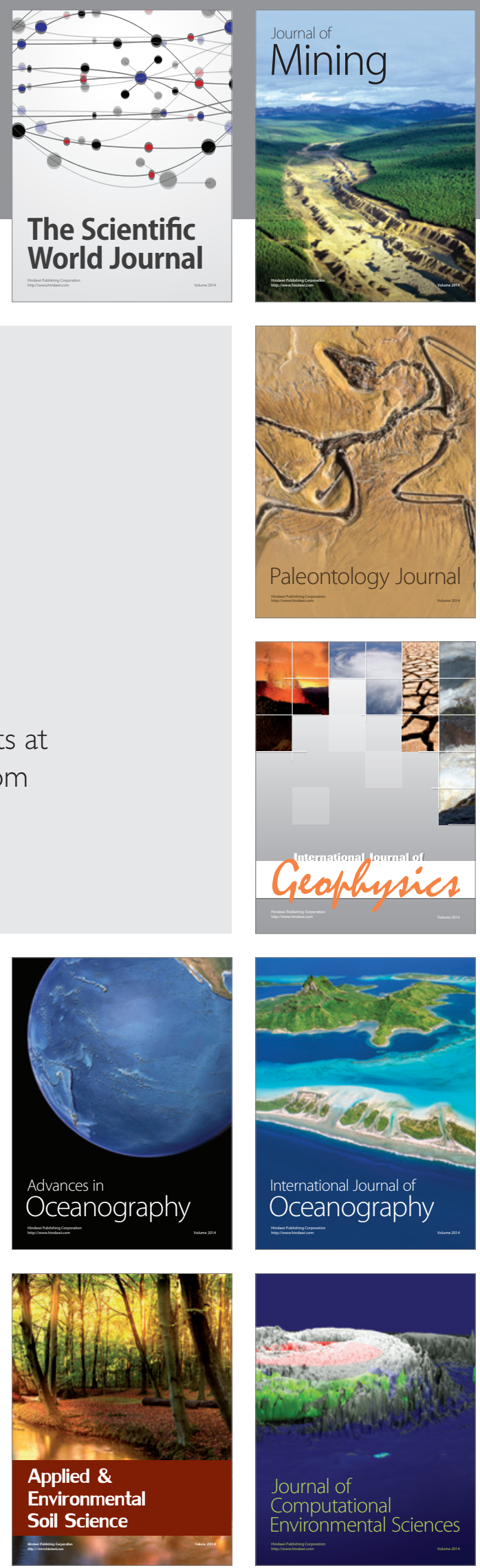